Check for updates

Cite this: RSC Adv., 2017, 7, 21323

Received 2nd February 2017

Accepted 9th April 2017

DOI: 10.1039/c7ra01380a

rsc.li/rsc-advances

\section{Alignment of Ge-imogolite nanotubes in isomalt with tunable inter-tube distances $\uparrow$}

\author{
Clement Levard, (D) *a Antoine Thill, ${ }^{\mathrm{b}}$ Astrid Avellan, ${ }^{\mathrm{a}}$ Cyprien Mauroy, ${ }^{\mathrm{a}}$ Vladimir Vidal, ${ }^{\mathrm{a}}$ \\ Andrea P. C. Campos, ${ }^{C}$ Armand Masion ${ }^{a}$ and Jerome Rose ${ }^{a}$
}

\begin{abstract}
This study shows the almost perfect alignment of inorganic nanotubes (Ge-imogolite) within polyol filaments. Alignment was obtained by simply cooling a Ge-imogolite-polyol mixture under mechanical stress. Additionally, inter-tube distances in the plane normal to the filaments can be tuned by simply varying the weight fraction of imogolite in the starting solution. The ease of the alignment protocol will stimulate interest for testing new applications which require a good control of the pore density and morphology (periodicity, and high monodispersity of the pore size distribution).
\end{abstract}

Aligning one-dimensional (1D) nanostructures whether organic or inorganic, has recently attracted much attention to develop innovative technological applications. ${ }^{1}$ Indeed, confinement inside nanotubes is likely to enhance existing reactivity or create new properties. For example, permeation rates of gas, water and protons inside carbon nanotubes (CNT) have been shown to exceed by orders of magnitude those observed for other nanopores of similar size. ${ }^{2}$ The origin of such improved transport properties was attributed to the ordering of the molecules under spatial confinement and hydrophobicity of the CNT walls. Remarkable optical properties can also derive from aligned 1D nanostructured objects. As an example, it has been shown that an organized array of CNT can act as a photonic band gap crystal. ${ }^{3}$ If carbon nanotubes remain the most publicized materials in the literature, much of the focus now shifts towards inorganic nanotubes. For example, very large, osmotically induced electric currents generated by salinity gradients were observed inside a single highly charged transmembrane boron nitride nanotube. ${ }^{4}$

Aligning anisotropic nanostructures often requires rather involved sample preparation procedures. ${ }^{1}$ In most cases, the orientation of the nanostructures along a given direction is achieved by applying an external force, such an electromagnetic field $^{5}$ or mechanical shear. ${ }^{6}$ The first approach is applicable only to materials with adequate electromagnetic properties. The application of mechanical shear however is not dependent on material properties other than its morphology. In all cases, the surrounding medium (e.g. solvent) needs to be sufficiently fluid to allow the movement of the nanostructures towards

${ }^{a}$ CEREGE, CNRS, Aix-Marseille Univ, IRD, UM34, UMR 7330, 13545 Aix en Provence, France.E-mail: levard@cerege.fr

${ }^{b}$ LIONS, NIMBE, CEA, CNRS, Université Paris-Saclay, 91191 Gif sur Yvette, France 'Aix-Marseille Université, CP2M, Faculté des Sciences, Campus de St Jérôme, F-13397 Marseille, France

$\dagger$ Electronic supplementary information (ESI) available. See DOI: 10.1039/c7ra01380a a preferential direction. This low viscosity of the matrix will cause the system to relax once the force used to create the alignment is no longer applied. A permanent alignment (outside the force field) thus requires immobilization of the nanostructures by changing the surrounding matrix and/or its properties.

Here we describe the successful alignment of inorganic nanotubes within an organic matrix. The tubes used in the present study were Ge-imogolite, i.e. the Ge analogues of natural imogolite, which are readily obtained by aqueous sol-gel processes with good control over tube length and diameter, wall multiplicity and chemistry; ${ }^{7-11}$ the matrix was isomalt, a sugar alcohol used in cooking.

The arrangement of the nanotubes within solidified isomalt filaments was explored as a function of initial Ge-imogolites concentration using Small Angle X-ray Scattering (SAXS) and Scanning Electron Microscopy (SEM).

\section{Ge-imogolite synthesis}

Ge-imogolite nanotubes were synthesized using a previously published protocol. ${ }^{9}$ Briefly, tetraethyl orthogermanate was added to an aluminum perchlorate solution. The $[\mathrm{Al}] /[\mathrm{Ge}]$ ratio was set to 2 with an initial aluminum concentration of $0.2 \mathrm{~mol}$ $\mathrm{L}^{-1}$. An urea solution at $0.2 \mathrm{~mol} \mathrm{~L}^{-1}$ was then added at room temperature to a ratio $R=$ [urea]/[Al] of 1 . Immediately after mixing, the solution was placed in an oven at $140{ }^{\circ} \mathrm{C}$ for 5 days. The solution was dialyzed against ultrapure water using a $8 \mathrm{kDa}$ membrane to eliminate residual salts and excess alcohol. The starting Ge-imogolite sample was characterized by atomic force microscopy (AFM) and small angle X-ray scattering (SAXS).

\section{Synthesis of the composite filaments}

Ge-imogolite-isomalt filaments were obtained by adapting a procedure used to align nanocellulose. ${ }^{12}$ Various quantities of 
Table 1 Initial masses of isomalt and Ge-imogolite and corresponding imogolite weight fraction $\left(f_{\mathrm{w}}\right) . q$ values of the first correlation peaks $\left(q_{\text {peak }}\right)$ and corresponding distance $\left(\langle D\rangle_{\text {exp }}\right)$. Orientational order parameter $\left(S_{0}\right)$

\begin{tabular}{llllll}
\hline & $m_{\text {isomalt }}(\mathrm{g})$ & $m_{\text {Ge-imogolite }}(\mathrm{g})$ & $f_{\mathrm{w}}$ & $q_{\text {peak }}\left(\AA^{-1}\right)$ & $\langle D\rangle_{\exp }(\AA)$ \\
\hline Filament \#1 & 0.5 & 0.0455 & 0.091 & 0.041 & 153 \\
Filament \#2 & 0.5 & 0.0325 & 0.065 & 0.035 & 180 \\
Filament \#3 & 0.5 & 0.0195 & 0.039 & 0.031 & 0.98 \\
Filament \#4 & 0.5 & 0.0065 & 0.013 & 0.021 & 203 \\
Filament \#5 & 0.5 & 0.00325 & 0.0065 & nd & 299 \\
\end{tabular}

Ge-imogolite solutions were mixed with $0.5 \mathrm{~g}$ of isomalt to vary the Ge-imogolite/isomalt ratio between $c a$. 0.6 and $9 \mathrm{wt} \%$ (Table 1). The solutions were placed in an oil bath and heated at $155^{\circ} \mathrm{C}$, i.e. just above the melting point of isomalt $\left(145-150{ }^{\circ} \mathrm{C}\right)$, to evaporate all water. Once all the water has evaporated, the obtained viscous nanotube dispersion was placed on a heating plate at $40{ }^{\circ} \mathrm{C}$. The Ge-imogolite/isomalt filaments were formed by dipping a spatula into the viscous suspension and pulling it up at a speed of $0.5 \mathrm{~m} \mathrm{~s}^{-1}$ until the filaments broke off due to cooling. A control sample without Ge-imogolite was also prepared.

\section{Ge-imogolite and filament characterization}

Tube length of Ge-imogolites stock solutions was measured using AFM (Innova Atomic Force Microscope, Bruker). Typical AFM images $(512 \times 512$ pixels $)$ were recorded in tapping mode with a pixel size of $5.85 \mathrm{~nm}$. A diluted drop of Ge-imogolite stock solution was deposited on a cleaved mica sheet and dried before measurement.

SAXS experiments were carried out laboratory scale instrumentation whose setup is detailed elsewhere. ${ }^{13}$ Two $q$ ranges were investigated depending on the information needed $(0.04<$ $q<1 \AA^{-1}$ for the characterization of the nanotube structures (stock solution) vs. $0.01<q<0.3 \AA^{-1}$ for the characterization of the nanotube inter-distances (within the filaments)). Stock solution was placed in a kapton capillary. Filaments were placed vertically in a sample holder. Scattered intensity $(I)$ as a function of the wave-vector modulus $(q)$ is obtained by angular integration over the recorded two-dimensional scattering pattern. In the case of the solution, the angular integration is performed on the whole image, while for the filaments, the intensity is averaged only in the preferential scattering direction.

SEM analysis was performed with a Zeiss GeminiSEM 500 ultra-high resolution FESEM at $1 \mathrm{kV}$. In-lens secondary electron detection was used for imaging. At $1 \mathrm{kV}$ the resolution is $1.1 \mathrm{~nm}$. For chemical analyses (Energy Dispersive X-ray Spectroscopy (EDS)), an EDAX Octane Silicon Drift Detector (129 eV energy resolution for Manganese) was used.

\section{Structural properties of Ge-imogolite}

The nanotube synthesis produced a Ge-imogolite suspension with a concentration of $6.5 \mathrm{~g} \mathrm{~L}^{-1}$ of Ge-imogolites (dry weight measurement). The SAXS signal of this suspension (Fig. 1) is characteristic of double-walled Ge-imogolite nanotubes. ${ }^{\mathbf{1 4}}$ The scattered intensity was fitted with a model consisting of two concentric $5 \AA$ thick cylinders (radii 20.8 and $12.0 \AA$ ) with $22 \mathrm{Ge}$ (electron density $\left.1.00 \mathrm{e}^{-} \AA^{-3}\right)$ and 12 Ge atoms $\left(0.96 \mathrm{e}^{-} \AA^{-3}\right)$ in the cross section for the outer and the inner tube respectively. The experimental intensity in absolute scale is fitted with a concentration of $7.0 \pm 0.7 \mathrm{~g} \mathrm{~L}^{-1}$ of imogolite which is
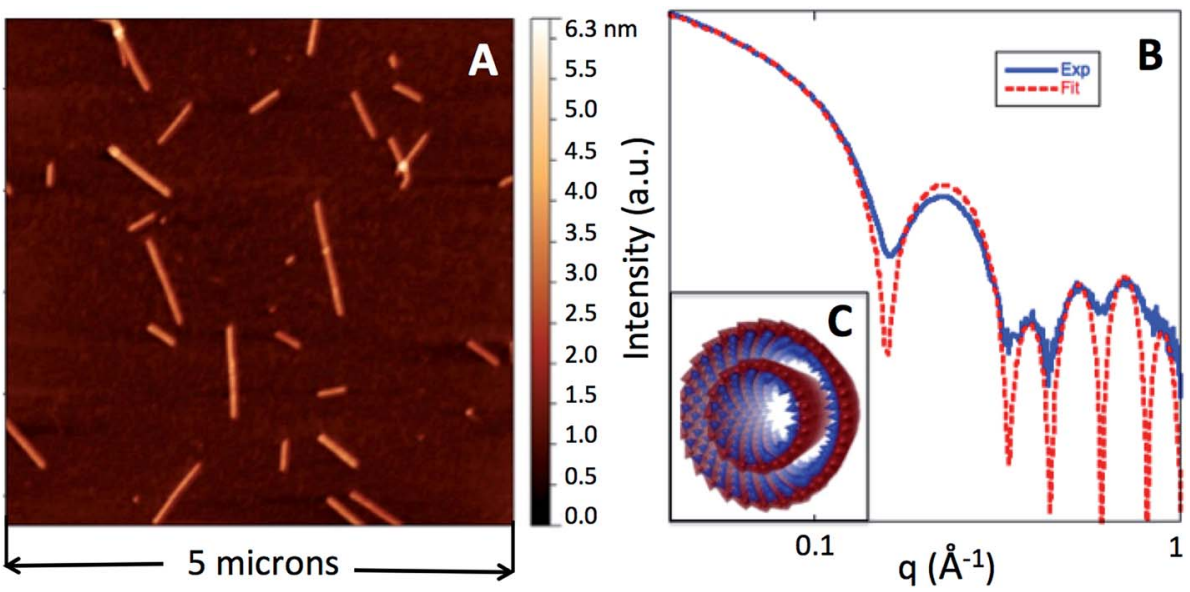

Fig. 1 AFM image of the Ge-imogolite nanotubes (A). Experimental (solid blue line) and calculated (dotted red line) /(q) curves (B). The calculated line corresponds to the form factor of two concentric homogeneous cylinders of radii 20.8 and $12.0 \AA$ and thickness $5 \AA$ (C). The electronic density of the homogeneous cylinders are 1.00 and $0.96 \mathrm{e}^{-} \AA^{-3}$ respectively. 
consistent with the above $6.5 \mathrm{~g} \mathrm{~L}^{-1}$ mass measurement. The corresponding volume fraction of Ge-imogolite nanotubes in the stock suspension is $0.2 \%$.

The typical tube length is beyond the accessible size range of the present SAXS instrument. The tube length distribution displays sizes from several tens of nanometers up to 1 micron with an average length of $190 \mathrm{~nm}$ (size distribution can be found elsewhere ${ }^{9}$ ). The large zeta potential, $50 \pm 5 \mathrm{mV}$, in the stock suspension (DI water, $\mathrm{pH}$ 6.5) is indicative of an $a$ priori strong electrostatic repulsion between the Ge-imogolite nanotubes.

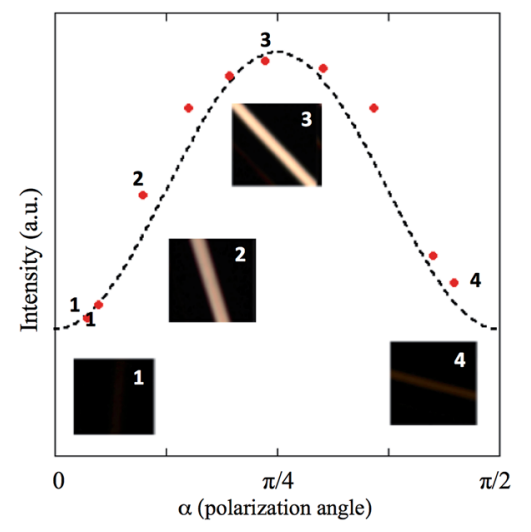

Fig. 2 Light intensity measured on filament \#3 as a function of the polarization angle. The dotted line corresponds to $I(\alpha) \sim \cos ^{2} \alpha \sin ^{2} \alpha$. For four of the data points (\#1-4) an image of filament \#3 is shown (image size is $1 \mathrm{~cm}^{2}$ ).

\section{Ge-imogolite alignment}

The arrangement of Ge-imogolite within the solidified isomalt matrix has been examined at several scales. At the largest scale, the Ge-imogolite-isomalt filaments were observed under crossed polarizers. At 0 and $\pi / 2 \mathrm{rad}$, no light is transmitted with filament \#3 (Fig. 2). For angles ( $\alpha$ ) between these values, light is transmitted through the sample. This is attributed to the Geimogolite nanotubes since no light transmission is detected for the control isomalt filament, regardless of the angle. For filament \#3, the measured intensities as a function of $\alpha$ display a pattern consistent with Malus' law, ${ }^{15}$ i.e. $I(\alpha) \sim \cos ^{2} \alpha \sin ^{2} \alpha$ (Fig. 2). This suggests the presence of an ordered structure along the length axis of the filament, i.e. an alignment of the Geimogolite nanotubes along this direction.

Further evidence for the ordered arrangement of $\mathrm{Ge}$ imogolite in the isomalt matrix is given by the strongly anisotropic X-ray scattering patterns (Fig. 3A), which is indicative of the alignment of the nanotubes along the axis of the filament. The corresponding SAXS curves of the imogoliteisomalt filaments (Fig. 3B) all display the characteristic oscillation centered at $0.22 \AA^{-1}$ already observed for Ge-imogolite in the stock solution (Fig. 1B). This shows that the structure of the tubes remains unaffected during the preparation of the filaments. The alignment of the Ge-imogolite nanotubes can be estimated quantitatively with the orientational order parameter $S_{\mathrm{o}}{ }^{16}$ The $S_{\mathrm{o}}$ factor was determined considering the Maier-Saupe orientational distribution using the following equation: ${ }^{16}$
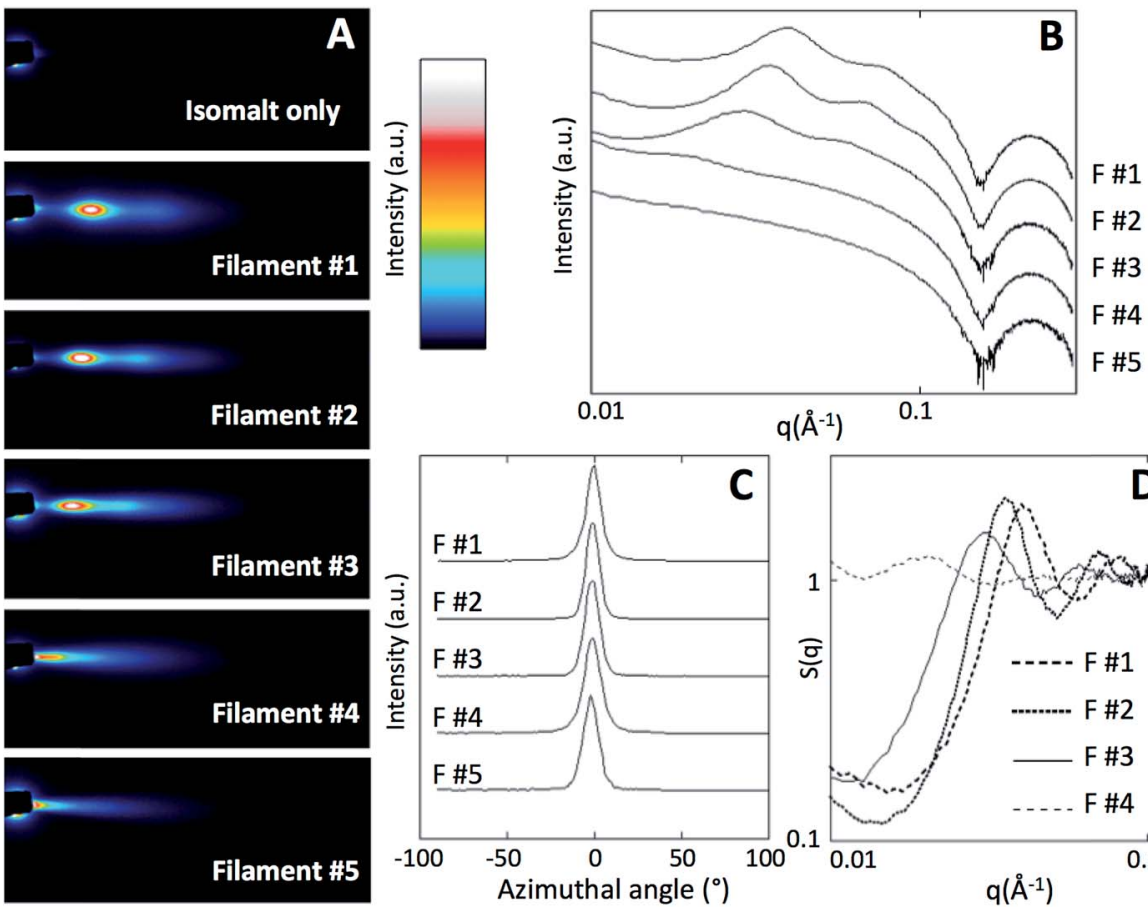

Fig. 3 X-ray scattering patterns for the control isomalt filament and the Ge-imogolite-isomalt composites (F\#1-F\#5) (dark squares top left corner: beam stop) (A). Corresponding /(q) and azimuthal curves for the Ge-imogolite/isomalt filaments (B and C respectively). S( $q$ ) curves for the Ge-imogolite/isomalt filaments (D). 


$$
S_{\mathrm{o}}=\frac{1}{2}\left\langle 3\left\langle\cos ^{2} \beta\right\rangle-1\right\rangle
$$

where $\beta$ is the deviation angle from the main orientation vector. The distribution of $\beta$ is obtained from the width of the scattered intensity versus azimuthal angle ${ }^{16}$ (Fig. 3C) (the azimuthal angle was chosen at the maximum peak intensity). As a general rule, $S_{\text {o }}$ increases with the level of orientation with values between 0 for an isotropic system and 1 for an unidirectional orientation of the objects $(\beta=0)$. For the filaments in the present study, the calculated $S_{\mathrm{o}}$ values between 0.97 and 0.99 (Table 1) show a nearly perfect alignment of the Ge-imogolite that does not depend on the weight fraction of the tubes. Earlier imogolite alignment experiments based on comparable, yet more elaborate, flow/shear protocols yielded poorer results with $S_{\mathrm{o}}$ around 0.6-0.7, ${ }^{6,17}$ which makes our easy-to-implement tube alignment procedure even more attractive.

\section{Arrangement of Ge-imogolite tubes within the isomalt matrix}

At the highest concentrations, the Ge-imogolite nanotubes are not distributed randomly within the matrix: the SAXS patterns for filaments \#1, \#2, \#3 display broad peaks (Fig. 3B) which are due to an ordered arrangement of the nanotubes in the plane normal to the filament axis. The associated structure factor $S(q)$ (Fig. 3D) clearly shows correlation peaks whose positions shift to higher $q$ with increasing concentration. The position of the first correlation peak is used to calculate an average inter-nanotube distance $\langle D\rangle_{\exp }$ (Table 1). As anticipated, the packing of the nanotubes becomes denser with increasing concentration. For $f_{\mathrm{w}}=0.039$ (filament \#4), the broadening of the correlation peak is probably indicative of a partial loss of the position correlation. For $f_{\mathrm{w}}=0.0065$ (filament \#5), no correlation peaks were observed, the Geimogolite concentration is too dilute and the position correlation is lost.

The ordered organization of the nanotubes observed at a semi-local scale was further confirmed at the local scale. Cross-sections of the Ge-imogolite nanotubes (bright dots) were clearly identified on the SEM image of the cross section of filament \#2, confirming the alignment of the nanotubes parallel to the filament axis. Those bright dots were not observed in the control filament (without Ge-imogolite) (Fig. S1†). Additionally, EDS analysis confirmed the presence of Ge and Al (Fig. S2, ESI $\dagger$ ). Additionally, Ge-imogolites were found to be well dispersed within the filament since no bundles were observed. Internanotube distances distribution was derived from the Fast Fourier Transform analysis of the SEM image (Fig. 4, inset). The inter-nanotube distance distribution, corresponding to the average radius of the bright ring, was found to be $19.5 \pm 1.5 \mathrm{~nm}$, i.e. close to the average distance measured by SAXS at the semilocal scale (18 $\mathrm{nm})$.

Alignment and even distribution of the Ge-imogolite tubes follows relatively fast kinetics, i.e. the few seconds between dipping the rod in the isomalt-nanotube suspension and the solidification of the filaments. This supports the hypothesis

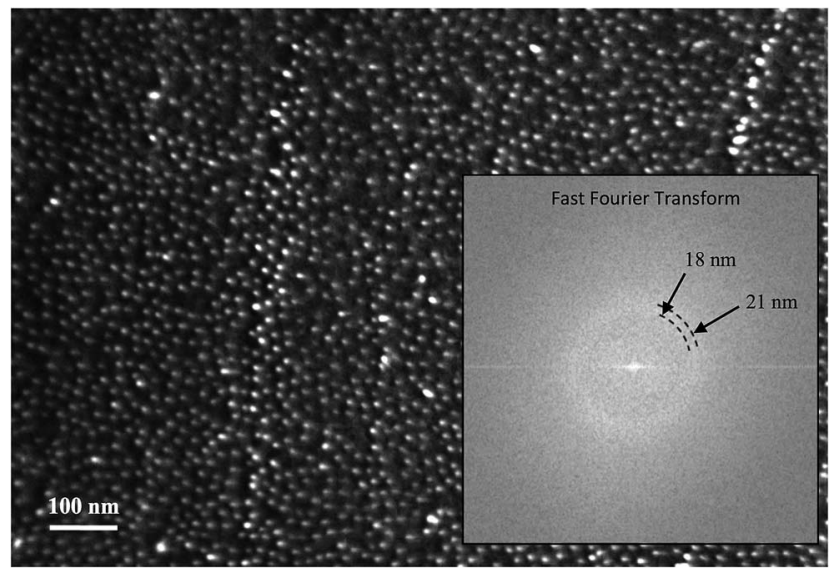

Fig. 4 SEM image of the cross-section of filament 2. Fast Fourier Transform of the SEM image is presented in the inset.

that strong electrostatic repulsions between the tubes are the main cause for their ordered arrangement. Additionally, one property of the matrix facilitates this process: isomalt is known to form an amorphous glass upon fast cooling, ${ }^{18,19}$ which means that the tubes have only to overcome the viscosity of the matrix as opposing force to their alignment and ordered packing. The formation of isomalt crystals which might interfere with the organization of the tubes occurs only at slow cooling rates, assuming that this crystallization remains unaffected by the presence of the Ge-imogolite tubes. Fast cooling of the filament under mechanical stress is thus a very simple method enabling a quasi-perfect alignment of the Geimogolite nanotubes along the filaments which make this system ideal for further investigations (e.g. testing the alignment and arrangement of tubes with various charges and aspect ratios); we believe that this study will stimulate the interest for the development of applied aspects which require a good control of the pore density and morphology (periodicity, no tortuosity and high monodispersity of the pore size distribution) such as optic systems among others (e.g. two dimensional photonic crystals or waveguides).

\section{Author contributions}

C. L. and A. T. designed the experiment. C. L., A. T., A. A. and C. M. performed the experiments. A P. C. C. performed the SEM analysis. All authors contributed to the data interpretation and wrote the manuscript.

\section{Acknowledgements}

The authors would like to warmly thank Hervé Bizot for fruitful discussion about the experimental setup. This work has been carried out in the framework of the Labex SERENADE (ANR-11LABX-0064) and of the A*MIDEX project (ANR-11-IDEX-000102), funded by the "Investissements d'Avenir" French Government program managed by the French National Research Agency (ANR). 


\section{References}

$1 \mathrm{~B} . \mathrm{Su}, \mathrm{Y}$. $\mathrm{Wu}$ and $\mathrm{L}$. Jiang, The art of aligning onedimensional (1D) nanostructures, Chem. Soc. Rev., 2012, 41(23), 7832-7856.

2 S. Guo, E. R. Meshot, T. Kuykendall, S. Cabrini and F. Fornasiero, Nanofluidic Transport through Isolated Carbon Nanotube Channels: Advances, Controversies, and Challenges, Adv. Mater., 2015, $27(38)$, 5726-5737.

3 K. Kempa, B. Kimball, J. Rybczynski, Z. P. Huang, P. F. Wu, D. Steeves, M. Sennett, M. Giersig, D. V. G. L. N. Rao, D. L. Carnahan, D. Z. Wang, J. Y. Lao, W. Z. Li and Z. F. Ren, Photonic Crystals Based on Periodic Arrays of Aligned Carbon Nanotubes, Nano Lett., 2003, 3(1), 13-18.

4 A. Siria, P. Poncharal, A. L. Biance, R. Fulcrand, X. Blase, S. T. Purcell and L. Bocquet, Giant osmotic energy conversion measured in a single transmembrane boron nitride nanotube, Nature, 2013, 494(7438), 455-458.

5 E. Paineau, M. E. M. Krapf, M.-S. Amara, N. V. Matskova, I. Dozov, S. Rouziere, A. Thill, P. Launois and P. Davidson, A liquid-crystalline hexagonal columnar phase in highlydilute suspensions of imogolite nanotubes, Nat. Commun., 2016, 7, 10271.

6 K. Kaneda, K. Uematsu, H. Masunaga, Y. Tominaga, K. Shigehara and K. Shikinaka, Flow-Orientation of Internal Structure and Anisotropic Properties on Hydrogels Consisted of Imogolite Hollow Nanofibers, Sen'i Gakkaishi, 2014, 70(7), 137-144.

7 C. Levard, J. Rose, A. Masion, E. Doelsch, D. Borschneck, L. Olivi, C. Dominici, O. Grauby, J. C. Woicik and J.-Y. Bottero, Synthesis of Large Quantities of Single-Walled Aluminogermanate Nanotube, J. Am. Chem. Soc., 2008, 130(18), 5862-5863.

8 C. Levard, E. Doelsch, J. Rose, A. Masion, I. Basile-Doelsch, O. Proux, J. L. Hazemann, D. Borschneck and J. Y. Bottero, Role of natural nanoparticles on the speciation of $\mathrm{Ni}$ in andosols of la Reunion, Geochim. Cosmochim. Acta, 2009, 73, 4750-4760.

9 M.-S. Amara, E. Paineau, M. Bacia-Verloop, M.-E. M. Krapf, P. Davidson, L. Belloni, C. Levard, J. Rose, P. Launois and
A. Thill, Single-step formation of micron long $(\mathrm{OH})_{3} \mathrm{Al}_{2} \mathrm{O}_{3} \mathrm{Ge}(\mathrm{OH})$ imogolite-like nanotubes, Chem. Commun., 2013, 49(96), 11284-11286.

10 A. Avellan, C. Levard, N. Kumar, J. Rose, L. Olivi, A. Thill, P. Chaurand, D. Borschneck and A. Masion, Structural incorporation of iron into Ge-imogolite nanotubes: a promising step for innovative nanomaterials, RSC Adv., 2014, 4(91), 49827-49830.

11 A. Avellan, C. Levard, C. Chaneac, D. Borschneck, F. R. A. Onofri, J. Rose and A. Masion, Accelerated microwave assisted synthesis of alumino-germanate imogolite nanotubes, RSC Adv., 2016, 6, 108146-108150.

$12 \mathrm{H}$. Bizot and B. Cathala, A route to uniaxially oriented ribbons of bacterial cellulose nanocrystals based on isomalt spun sacrificial template, Nord. Pulp Pap. Res. J., 2014, 29(1), 15-18.

13 O. Taché, S. Rouzière, P. Joly, M. S. Amara, B. Fleury, A. Thill, P. Launois, O. Spalla and B. Abécassis, MOMAC: A SAXS/ WAXS laboratory instrument dedicated to nanomaterials, $J$. Appl. Crystallogr., 2016, 49, 1624-1631.

14 P. Maillet, C. Levard, E. Larquet, C. Mariet, O. Spalla, N. Menguy, A. Masion, E. Doelsch, J. Rose and A. Thill, Evidence of Double-Walled Al-Ge Imogolite-Like Nanotubes. A Cryo-TEM and SAXS Investigation, J. Am. Chem. Soc., 2010, 132, 1208-1209.

15 G. Bruhat and A. Kastler, Optique, in Cours de physique générale, Masson, Paris, 6th edn, 1992.

16 P. Davidson, D. Petermann and A. M. Levelut, The measurement of the nematic order parameter by X-ray scattering reconsidered, J. Phys. II, 1995, 5(1), 113-131.

17 K. Shikinaka, Y. Koizumi, K. Kaneda, Y. Osada, H. Masunaga and K. Shigehara, Strain-induced reversible isotropicanisotropic structural transition of imogolite hydrogels, Polymer, 2013, 54(10), 2489-2492.

18 H. K. Cammenga and B. Zielasko, Thermal behaviour of isomalt, Thermochim. Acta, 1996, 271, 149-153.

19 H. K. Cammenga and B. Zielasko, Glasses of sugars and sugar substitutes, Ber. Bunsen-Ges. Phys. Chem., 1996, 100(9), 1607-1609. 